\title{
Weyl Semimetal Path to Valley Filtering in Graphene
}

\author{
Ahmed M. Khalifa, ${ }^{1}$ Ribhu K. Kaul, ${ }^{1}$ Efrat Shimshoni, ${ }^{2}$ H.A. Fertig, ${ }^{3}$ and Ganpathy Murthy ${ }^{1}$ \\ ${ }^{1}$ Department of Physics and Astronomy, University of Kentucky, Lexington, KY 40506-0055 \\ ${ }^{2}$ Department of Physics, Bar-Ilan University, Ramat-Gan 52900, Israel \\ ${ }^{3}$ Department of Physics, Indiana University, Bloomington, IN 47405
}

\begin{abstract}
We propose a device in which a sheet of graphene is coupled to a Weyl semimetal, allowing for the physical access to the study of tunneling from two-dimensional to three dimensional massless Dirac fermions. Due to the reconstructed band structure, we find that this device acts as a robust valley filter for electrons in the graphene sheet. We show that, by appropriate alignment, the Weyl semimetal draws away current in one of the two graphene valleys while allowing current in the other to pass unimpeded. In contrast to other proposed valley filters, the mechanism of our proposed device occurs in the bulk of the graphene sheet, obviating the need for carefully shaped edges or dimensions.
\end{abstract}

Weyl semimetals (WSMs) [1-5] are three-dimensional materials with an even number of isolated band touching points in the Brillouin zone (BZ) called Weyl nodes. The band dispersion near each Weyl node is that of a massless Weyl fermion, which is chiral, the chirality being encoded in the Berry flux pierced by a surface in momentum space enclosing the Weyl node. Either inversion [1] or timereveral symmetry $[2-5]$ or both must be broken in WSMs. Many examples of WSM materials are now known [611]. In particular, one recent material, $\mathrm{Co}_{3} \mathrm{Sn}_{2} \mathrm{~S}_{2},[12,13]$ breaks both time-reversal and inversion, and possesses cleaved surfaces with three-fold symmetry.

In monolayer graphene (MLG) [14] electrons near charge neutrality belong to one of the two Dirac points ( $\mathbf{K}$ and $\mathbf{K}^{\prime}$, related to each other by inversion and timereversal) which constitute valleys. Due to the large difference in lattice momentum, the valley degree of freedom is highly conserved in transport. This has made it a promising material for use in valleytronics, which seeks to use the valley degree of freedom to encode and manipulate information [15]. Either electrons or excitons can be used to encode information; in the following we will focus on electrons. A necessary first step in this valleytronics program in MLG is to be able to produce valley-polarized current, usually done by valleyfiltering an incident valley-unpolarized current. There are many theoretical proposals for doing so. Methods preserving the time-reversal of MLG while breaking inversion [16] include a constriction with tailored edges [17], using the "high-energy" dispersion of electrons away from the Dirac points [18], using strain, which creates an internal gauge field acting oppositely on the two valleys to spatially separate valley currents [19], lattice defects [20] and spin-orbit coupling [21]. Methods that break time-reversal include the use of magnetic and potential barriers [22], or tunnel-coupling monolayer and bilayer graphene with an in-plane magnetic field to tune momentum [23]. Yet other proposals include using adiabatic pumping [24] or Floquet methods [25, 26] to separate the valleys. Most of the proposals need precise control of edges/strain/substrates/superlattices, and/or

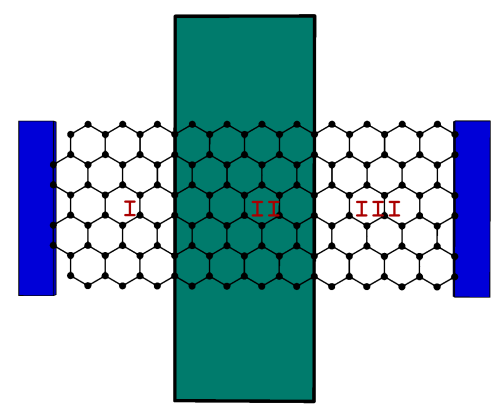

FIG. 1: A schematic picture for a graphene/WSM device. The incoming current (region I) is equally populated in the two valleys and the outgoing current (region III) is valley polarized.

depend very sensitively on the energy of the electrons to be valley-filtered.

In this work we show that the surface of a WSM with three-fold symmetry, breaking both time-reversal and inversion, is a natural substrate for robust valley-filtering current in MLG (see Fig. 1 for the proposed device). The minimal number of Weyl nodes is six, as seems to be the case for $\mathrm{Co}_{3} \mathrm{Sn}_{2} \mathrm{~S}_{2}$. When the chemical potential is at the energy of the Weyl nodes, their projections on the surface BZ (also three-fold symmetric) are points, connected by zero energy surface Fermi arc (FA) states (see Fig. 2(a)). Upon doping, the projection of the bulk states at fixed energy on to the surface BZ will be solid regions enclosing the Weyl point projections (WPPs), as shown in Figs. 2(b), 2(c). We refer to these solid regions as "Fermi pockets." We emphasize that: (i) The Fermi pockets break inversion symmetry. (ii) Each $\vec{k}$ in the surface BZ has a continuum of bulk states of the WSM projected on to it. The next step is to weakly tunnel-couple the MLG to the surface of the WSM in their region of overlap, taking care to align it so that the $\mathbf{K}$ Dirac point lies within a Fermi pocket in the surface BZ of the WSM, up to a reciprocal lattice vector of the surface $\mathrm{BZ}$ of the WSM, as shown in Fig. 2(d). We emphasize that the $\mathbf{K}^{\prime}$ 


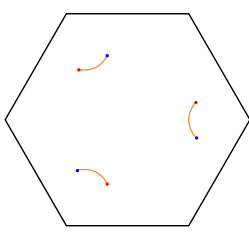

(a)

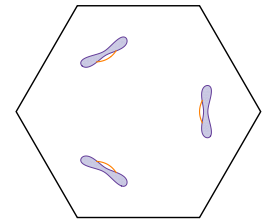

(b)

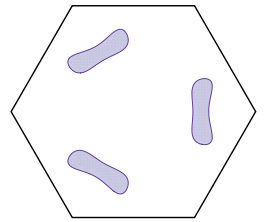

(c)

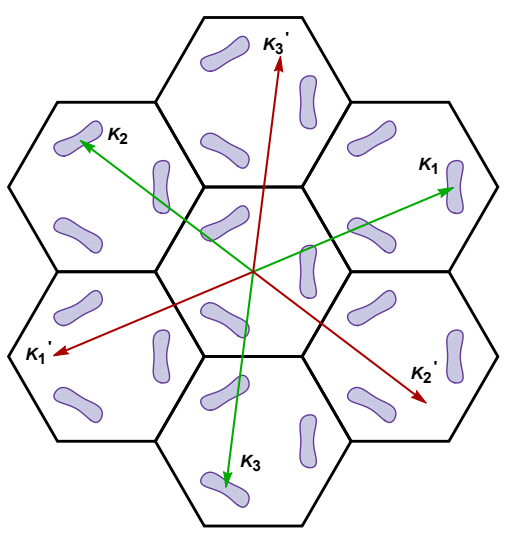

(d)

FIG. 2: Evolution of the projected WSM states in the SBZ. (a) At the nodal energy $\left(E_{F}=0\right)$, only the Fermi arcs are present in the SBZ. (b),(c) As the chemical potential is raised, the Fermi arcs get absorbed into the bulk states and eventually disappear in (c). (d) An incommensurate structure of graphene and the WSM shown in momentum space. The green vectors show the three equivalent graphene $\mathbf{K}$ points. For a range of twist angles, the low-energy states in the $\mathbf{K}$ valley of graphene lie in a band of the projected bulk states of the WSM. The graphene states near the $\mathbf{K}^{\prime}$ points (red vectors) do not overlap the projected bulk states of the

WSM in energy, but instead lie in a band gap.

point does not overlap a Fermi pocket.

Consider a current injected into the graphene sheet from the left, in region I of the device depicted in Fig. 1. Before it enters region II, where the MLG and the WSM are tunnel-coupled, the current is divided equally between the two valleys. When it enters region II, each electronic state in the $\mathbf{K}$ valley is coupled to (and lies in the middle of) a band of bulk states in the WSM. We assume, plausibly, that the Fermi velocity of graphene is much higher than that of the WSM, implying that all states near the chemical potential $\mu$ of the MLG will lie in the middle of the bulk band of the WSM. Each state in the MLG $\mathbf{K}$ valley will therefore hybridize with them and broaden, resulting in a decay of the current in the $\mathbf{K}$ valley into the bulk of the WSM, which is assumed to be grounded. By contrast, although the $\mathbf{K}^{\prime}$ valley band structure is modified by tunnel-coupling to the WSM, there are no bulk or surface states of the WSM at the same energy, so that the current in this valley will suffer at most a finite diminution due to reflections at the various interfaces of the structure. Note that the greater the length of the tunnel-coupled region, the greater the degree of valley polarization of the outgoing current.

A few remarks are in order about the generality and robustness of our proposal. (i) Without additional symmetries, there is no reason for the chemical potential in the WSM to lie at the Weyl point energy. Thus, generically, the WSM will have Fermi pockets at the surface. Indeed this seems to be the case for $\mathrm{Co}_{3} \mathrm{Sn}_{2} \mathrm{~S}_{2}$ [12]. (ii) This implies that the alignment of the MLG on the WSM surface can be varied over a range of angles while maintaining the condition that $\mathbf{K}$ sits within a Fermi pocket, while $\mathbf{K}^{\prime}$ does not. Thus, fine-tuning the alignment of graphene on the WSM surface is not necessary. (iii) Scanning the chemical potential can be achieved by doping the WSM and/or gating the MLG. Our proposal will work over a wide range of electron energies near charge neutrality in MLG. (iv) The details of the tunneling matrix elements between the MLG and the surface of the WSM are irrelevant: what matters is that each $\vec{k}$ state within the $\mathbf{K}$ valley is coupled to the WSM continuum. (v) Smooth disorder in the WSM or MLG will scatter single-particle states close in momenta. Since $\mathbf{K}$ and $\mathbf{K}^{\prime}$ are far apart, the valley-filtering will be robust against smooth disorder.

Having established the generality and wide applicability of our proposal, in the remainder of this paper we analyze a specific model of such a device, illustrating the physical behaviors described above. In order to treat arbitrary tunnel coupling strengths via tight-binding, we construct a simple model of the three-fold symmetric WSM, and assume that its surface is commensurate with that of MLG. We treat only the simplest and most symmetric case in the main text, leaving the general case to the supplemental material [30].

WSM model.- Our starting point is a minimal twoband model for a Weyl semimetal on a triangular lattice which breaks both time reversal and inversion symmetry, but possesses three-fold rotational symmetry. In momentum space, the Hamiltonian is given by

$$
H\left(\vec{k}, k_{z}\right)=\sum_{\mu=x, y, z} f_{\mu} \sigma_{\mu}
$$

where $f_{x}=2 t\left[1-\cos \left(k_{z}\right)+\mu_{1}-\sum_{i=1}^{3} \cos \left(\vec{k} \cdot \overrightarrow{a_{i}}\right)\right], f_{y}=$ $2 t\left[\sum_{i=1}^{3} \sin \left(\vec{k} \cdot \overrightarrow{a_{i}}\right)-\mu_{2}\right]$ and $f_{z}=2 t^{\prime} \sin \left(k_{z}\right)$. Note that $\vec{k}$ here is a two dimensional vector and $\sigma_{\mu}$ are the usual Pauli spin matrices, and $t$ and $t^{\prime}$ represent the in-plane and out-of-plane hoppings, respectively. The three $\vec{a}_{i}$ vectors are the nearest-neigbor vectors on the triangular lattice, $\vec{a}_{1}=a \hat{x}$ and $\vec{a}_{2,3}=a\left(\frac{-1}{2} \hat{x} \pm \frac{\sqrt{3}}{2} \hat{y}\right)$. The 3 -fold rotational symmetry of $H$ is manifested in its en- 
ergy spectrum. The band structure possesses three pairs of Weyl nodes related to one another by 3 -fold rotations. These are found at $k_{z}=0$, with $\vec{k}$ satisfying $\mu_{1}-\sum_{i=1}^{3} \cos \left(\vec{k} \cdot \overrightarrow{a_{i}}\right)=0$ and $\sum_{i=1}^{3} \sin \left(\vec{k} \cdot \overrightarrow{a_{i}}\right)-\mu_{2}=0$. The positions of the Weyl nodes can be moved by varying $\mu_{1}$ and $\mu_{2}$. We assume that the free surface of the WSM is in the $x y$ plane, which has three-fold symmetry. The Weyl point projections (WPPs) on to the surface Brillouin zone (SBZ) are connected by Fermi arcs. By standard methods [27] we find the energy dispersion for the Fermi arc states to be

$$
E=2\left[\sum_{i=1}^{3} \sin \left(\vec{k} \cdot \overrightarrow{a_{i}}\right)-\mu_{2}\right]
$$

$M L G$ commensurate with the surface of the WSM.- We adopt a model in which the MLG lattice is commensurate with that of the surface of the WSM, and that the WSM lattice constant is smaller than that of the MLG. While these assumptions are unrealistic for real materials, they allow us to use the full power of translation invariance to do nonperturbative calculations in the tunnel-couplings without fundamentally changing the character of the system. Calculations for incommensurate lattices are necessarily either perturbative in the tunnel-couplings, or dependent on truncations in momentum space [28, 29], both of which we wish to avoid. We emphasize that our proposal for valley-filtering does not depend on the commensuration we assume for our concrete model.

A schematic picture of our commensurate model is shown in Fig. 3. In order to study the electronic prop-

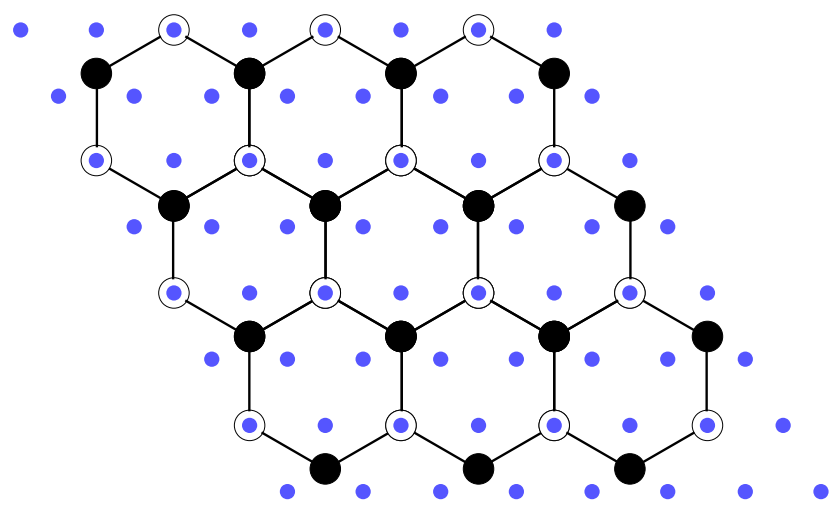

FIG. 3: Graphene and the top surface of our WSM model in a commensurate alignment which we use in our numerical tight binding calculation. Electrons on the $A$ sublattice of MLG (empty circles) are allowed to hop only to the WSM surface site they overlie, while electrons on the $B$ sublattice (solid black) of MLG can hop to the three WSM surface sites surrounding the given $B$ site.

erties of this system, we take a finite slab of the WSM in Eq. (1) along the $z$ axis and assume the system to be translationally invariant in the $x y$ plane. Going to real space in the $z$ direction in Eq. (1), we obtain the WSM slab Hamiltonian

$$
\begin{aligned}
H_{W S M} & =\sum_{n=0}^{N} \sum_{\vec{k}}\left[C_{n}^{\dagger}(\vec{k}) M(\vec{k}) C_{n}(\vec{k})-C_{n+1}^{\dagger}(\vec{k}) T C_{n}(\vec{k})\right. \\
& \left.-C_{n}^{\dagger}(\vec{k}) T^{\dagger} C_{n+1}(\vec{k})\right],
\end{aligned}
$$

where $C_{n}(\vec{k})$ is a two-component annihilation operator indexed by layer $n$ and

$$
\begin{aligned}
M(\vec{k}) & =2\left[\left(1+\mu_{1}-\sum_{i} \cos \left(\vec{k} \cdot \overrightarrow{a_{i}}\right)\right) \sigma_{x}\right. \\
& \left.+\left(\sum_{i} \sin \left(\vec{k} \cdot \overrightarrow{a_{i}}\right)-\mu_{2}\right) \sigma_{z}\right] .
\end{aligned}
$$

Spin has been suppressed for notational convenience. Note that $T=\sigma_{x}+i t^{\prime} \sigma_{y}, N$ is the thickness of the slab, and that we have set the hopping in the plane our energy unit, $t=1$. The total Hamiltonian is

$$
H=H_{W S M}+H_{G}+H_{t},
$$

where $H_{G}$ is the nearest neighbor hopping Hamiltonian of MLG. $H_{t}$ allows electrons in MLG to tunnnel to the top layer of the WSM in a translation-invariant way.

$$
H_{t}=\sum_{\vec{R}} \sum_{\vec{r}}\left[C_{0}^{\dagger}(\vec{r}) V_{\alpha}(|\vec{r}-\vec{R}|) f_{\alpha}(\vec{R})+h . c\right],
$$

where $C_{0}(\vec{r})$ is the two-component annihilation operator on the top $n=0$ layer of the WSM at site $\vec{r}$, and $f_{\alpha}(\vec{R})$ is an annihilation operator on the sublattice $\alpha=A, B$ at site $\vec{R}$ in graphene.

To ensure our requirement that the neighborhood of the $\mathbf{K}$ point of MLG lies within a bulk band of energies of the WSM we assume that the $\mathbf{K}$ point of MLG lies on a Fermi arc. From Eq. (2), this is achieved when $\mu_{2}=-\sqrt{3} / 2$. The $\mathbf{K}^{\prime}$ point will then reside in the gap of the WSM. We then diagonalize Eq. (4) for this value of $\mu_{2}$ to get the band structure of the system. We restrict ourselves to nearest-neighbor hopping only in $H_{t}$. This operationally means that electrons on the $A$ sublattice of MLG hop only to the WSM surface site at the same $x y$ coordinates with a spin-independent amplitude $\kappa$, while electrons on the $B$ sublattice of MLG can hop to the three sites of the WSM surface surrounding it with spin-independent amplitude $\kappa^{\prime}$. As we show in the supplemental material [30], moving the Fermi arcs or making the hopping more generic does not make any qualitative difference to our results.

Our tight-binding calculations presented in Fig. 4(a),(b) are consistent with expectations from the generic 

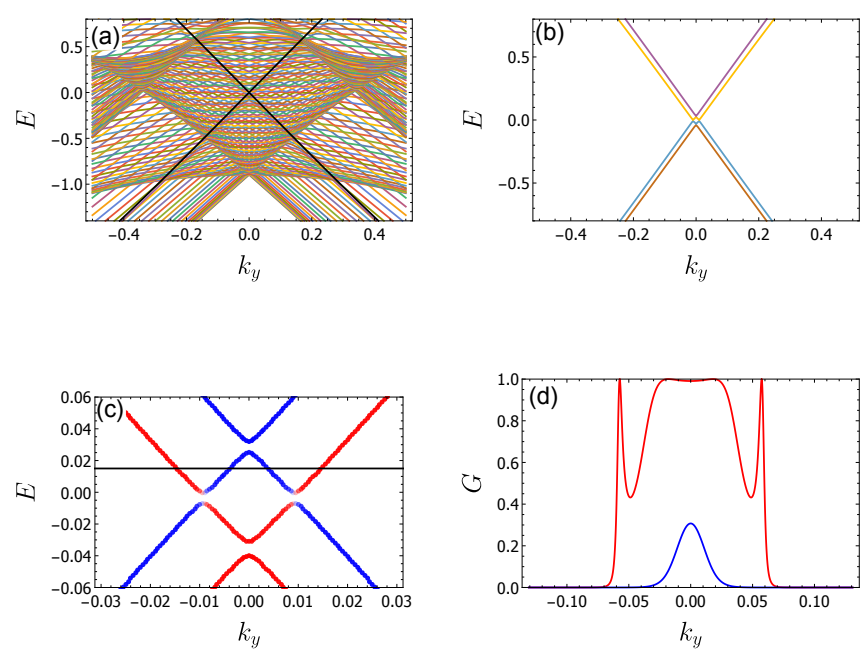

FIG. 4: Results of the tight binding calculation. (a),(b) The graphene/WSM spectrum is shown near the $\mathbf{K}$ and the $\mathbf{K}^{\prime}$ valleys, respectively. The Dirac cone in the $\mathbf{K}$ valley (shown in black) is immersed in the bulk states of the WSM while the Dirac cone near the $\mathbf{K}^{\prime}$ valley is slightly perturbed. (c) An expanded view zooming in on the $\mathbf{K}^{\prime}$ valley shows an inverted band structure where the colors code the average of $S_{z}$ operator with red being spin up and blue spin down. (d) The conductance of the graphene/WSM device based on a simplified model for the states near the $\mathbf{K}^{\prime}$ valley is shown in units of $e^{2} / h$. The conductance shows partial spin polarization as red denotes spin up states where blue denotes spin down states.

incommensurate case discussed earlier. We see that the Dirac cone for the $\mathbf{K}$ valley is immersed in the continuum of the bulk states while the $\mathbf{K}^{\prime}$ valley is isolated in the gap. A close examination of Fig. 4(b) reveals an induced spin-orbit (SO) coupling in the states of graphene's $\mathbf{K}^{\prime}$ valley.

The tight-binding results show that, at the $\mathbf{K}^{\prime}$ valley, the bands split into (almost purely) $\uparrow$ and $\downarrow$ bands with a band inversion near the $\mathbf{K}^{\prime}$ point (Fig. 4(c)). This can lead to interesting consequences for the transport: the different sizes of the Fermi surfaces for the two spin species (at the Fermi level marked black in Fig. 4(c)) will lead to different transmission probabilities, and consequently a (partial) spin polarization of the fully valley polarized current.

A simple model that reproduces the tight-binding results is the following effective Hamiltonian:

$$
H_{e f f}(\vec{k})=-k_{x} \tau_{x}+k_{y} \tau_{y}+\lambda_{1} \tau_{z}-\lambda_{2} \sigma_{z},
$$

where $\tau$ and $\sigma$ denote Pauli matrices corresponding to the sublattice and spin degrees of freedom, respectively, while $\lambda_{1}$ and $\lambda_{2}$ are the sublattice- and time-reversal-breaking parameters induced by integrating out the gapped WSM states.

Fig. 4(d) shows the Landauer conductance $\left(G=\frac{e^{2}}{h} T\right.$, where $T$ is the transmission probability; see the Supplemental Material [30] for details) of our proposed device (Fig. 1) in the two spin channels. We have assumed that there is translation invariance in the $y$-direction, perpendicular to the current flow. Given our assumptions, the conductance is due to the $\mathbf{K}^{\prime}$ valley only. Since the coupling to the WSM breaks both the sublattice and timereversal symmetries of MLG, it is natural that the conductances in the two spin channels are unequal.

Conclusions.-We have shown that overlaying graphene on a 3 -fold symmetric surface of a WSM breaking both time-reversal and inversion, with an alignment which places the $\mathbf{K}$ point of MLG in a Fermi pocket of the surface BZ of the WSM (modulo reciprocal lattice vectors of the WSM), will lead to a robust valley filter for graphene.

The physics leading to this may be stated concisely: states near the $\mathbf{K}$ valley of MLG lie within a band of bulk states of the WSM, projected to the surface BZ, hybridizing with the bulk states and "dissolving" into them. Current-carrying electrons in the $\mathbf{K}$ valley will scatter into bulk states of the (grounded) WSM, carrying them away from the MLG layer. States near the $\mathbf{K}^{\prime}$ valley, on the other hand, lie in a bandgap of the WSM, and will remain localized in the MLG, though their transport will be modified by the sublattice and time-reversal breaking induced by the WSM. Thus, for a sufficiently long interface (along the current direction), only the current in the $\mathbf{K}^{\prime}$ valley survives. This current is expected to have a nonzero spin polarization, whose precise value depends on the details of the interface coupling.

Our proposal does not require precise alignment between graphene and the surface of the WSM, precise control of the tunneling at the interface or the chemical potential of the current-carrying electrons. Smooth disorder will not degrade the valley-filtering. Upon doping the WSM appropriately, our proposal will work for Bernalstacked/twisted bilayer graphene [14] and transitionmetal-dichalcogenides [31] as well.

Diverse applications in addition to valley-filtering can also be considered. With small changes, the WSM could be used as a contact that is electrically connected only to one valley, which could be used to probe equilibrium correlated states in the quantum Hall regime of MLG. It would also be interesting to ask how the correlated states in magic-angle twisted bilayer graphene [32] respond when the states near one valley dissolve into the WSM bulk. We hope to address these and other questions in the near future.

We acknowledge support from the National Science Foundation via grant nos. DMR-2026947 (A.M.K and R.K.K.), ECCS-1936406 and DMR-1914451 (H.A.F.), as well as the support of the Research Corporation for 
Science Advancement through a Cottrell SEED Award (H.A.F); the US-Israel Binational Science Foundation through awards No. 2016130 (H.A.F, G.M. and E.S.) and 2018726 (H.A.F and E.S.); and the Israel Science Foundation (ISF) Grant No. 993/19 (E.S.).

[1] S. Murakami, New J. Phys. 9, 356 (2007).

[2] X. Wan, A. M. Turner, A. Vishwanath and S. Y. Savrasov, Phys. Rev. B 83, 205101 (2011); P. Hosur, S. A. Parameswaran and A. Vishwanath, Phys. Rev. Lett. 108, 046602 (2012).

[3] K. Y. Yang, Y. M. Lu and Y. Ran, Phys. Rev. B 84, 075129 (2011).

[4] A. A. Burkov and L. Balents, Phys. Rev. Lett. 107, 127205 (2011).

[5] G. Xu, H. Weng, Z. Wang, X. Dai, and Z. Fang, Phys. Rev. Lett. 107, 186806 (2011).

[6] B. Q. Lv, H. M. weng, B. B. Fu, X. P. Wang, H. Miao, J. Ma, P. Richard, X. C. Huang, L. X. Zhao, G. F. Chen et al, Phys. Rev. X 5, 031013 (2015).

[7] S.-Y. Xu, I. Beloploski, N. Alidoust, M. Neupane, G. Bian, C. Zhang, R. Sankar, G. Chang, Z, Yuan, C.-C. Lee et al, Science 349, 613 (2015).

[8] S.-Y. Xu, N. Alidoust, I. Blopolski, Z. Yuan, G. Bian, T.-R. Chang, H. Zheng, V. N. Strocov, D. S. Sanchez, G. Chang et al, Nat. Phys. 11, 748 (2015).

[9] H. Inoue, A. Gyenis, Z. Wang, J. Li, S. W. Oh, S. Jiang, N. Ni, B. A. Bernevig, and A. Yazdani, Science 351, 1184 (2016).

[10] E. H. Silva Neto, Science 365, 1248 (2019).

[11] I. Belopolski, K. Manna, D. S. Sanchez, G. Chang, B. Ernst, J. Yin, S. S. Zhang, T. Cochran, N. Shumiya, H. Zheng et al, Science 365, 1278 (2019).

[12] D. F. Liu, A. J. Liang, E. K. Liu, Q. N. Xu, Y. W. Li, C. Chen, D. Pei, W. J. Shi, S. K. Mo, P. Dudin et al, Science 365, 1282 (2019).

[13] N. Morali, R. Batabyal, P. K. Nag, E. Liu, Q. Xu, Y. Sun, B. Yan, C. Felser, N. Avraham, and H. Beidenkopf, Science 365, 1286 (2019).

[14] A. H. Castro Neto, F. Guinea, N. M. R. Peres, K. S. Novoselov, and A. K. Geim, Rev. Mod. Phys. 81, 109 (2009).

[15] J. R. Schaibley, H. Yu, G. Clark, P. Rivera, J. S. Ross, K. L. Seyler, W. Yao, and X. Xu, Nature Reviews Materials 1, 16055 (2016).

[16] D. Xiao, W. Yao, and Q. Niu, Phys. Rev. Lett. 99, 236809 (2007).

[17] A. Rycerz, J. Tworzydlo, and C. W. J. Beenakker, Nature Physics 3, 172 (2007).
[18] J. L. Garcia-Pomar, A. Cortijo, and M. Nieto-Vesperinas, Phys. Rev. Lett. 100, 236801 (2008); Y. S. Ang, S. A. Yang, C. Zhang, Z. Ma, and L. K. Ang, Phys. Rev. B. 96, 245410 (2017).

[19] Z.-P, Niu, Jour. Appl. Phys. 111, 103712 (2012); S. P. Milovanovic and F. M. Peeters, Appl. Phys. Lett. 109, 203108 (2016); M. Settnes, S. R. Power, M. Brandbyge, and A.-P. Jauho, Phys. Rev. Lett. 117, 276801 (2016); T. Stegmann and N. Szpak, 2D Materials 6, 015024 (2019).

[20] D. Gunlycke and C. T. White, Phys. Rev. Lett. 106 , 136806 (2011); L. H. Ingaramo and L. E. F. Foa Torres, J. Phys. Condens. Matter 28, 485302 (2016).

[21] M. M. Grujic, M. Z. Tadic, and F. M. Peeters, Phys. Rev. Lett. 113, 046601 (2014).

[22] D. Moldovan, M. Ramezani Masir, L. Covaci, and F. M. Peeters, Phys. Rev. B 86, 115431 (2012); F. Zhai, Y. Ma, and Y.-T. Zhang, JPCM 23, 385302 (2011); F. Zhai, Nanoscale 4, 6527 (2012); F. Zhai and K. Chang, Phys. Rev. B 85, 155415 (2012); Y. Wang, J. App. Phys. 114, 073709 (2013); W.-T. Lu, Phys. Rev. B 94, 085403 (2016); M. Settnes, J. H. Garcia, and S. Roche, 2D Materials 4, 031006 (2017); J. Wang, M. Long, W.-S. Zhao, G. Wang, and K. S. Chan, J. Phys. Condens. Matter 28, 285302(2016); Q.-P. Wu, Z.-F. Liu, A.-X. Chen, X.-B. Xiao, and Z.-M. Liu, Scientific Reports 6, 21950 (2016); M. M. Asmar and S. E. Ulloa, Phys. Rev. B 96, 201407(R) (2017); A. R. S. Lins and J. R. F. Lima, Carbon 160, 353 (2020); Q.-P. Wu, L.-L. Chang, Y.-Z. Li, X.-B. Xiao, Z.-F. Liu, Physica E 118, 113864 (2020).

[23] L. Pratley and U. Zulicke, App. Phys. Lett. 104, 082401 (2014).

[24] Y. Jiang, T. Low, K. Chang, M. I. Katsnelson, and F. Guinea, Phys. Rev. Lett. 110 (2013).

[25] F. Qi and G. Jin, J. App. Phys. 115, 173701 (2014).

[26] A. Kundu, , H.A. Fertig, and B. Seradjeh, Phys. Rev. Lett. 116016802 (2016).

[27] G. Murthy, H. A. Fertig, and E. Shimshoni, Phys. Rev. Research 2, 013367(2020).

[28] R. Bistritzer and A. H. MacDonald, Proc. Nat. Acad. Sci. USA 108, 12233 (2011).

[29] P. San-Jose, J. Gonzalez, and F. Guinea, Phys. Rev. Lett.108, 216802 (2012).

[30] Supplementary Material.

[31] For a review, see, S. Manzeli, D. Ovchinnikov, D. Pasquier, O. V. Yazyev, and A. Kis, Nat. Rev. Mater. 2, 17033 (2017).

[32] Y. Cao, V. Fatemi, S. Fang, K. Watanabe, T. Taniguchi, E. Kaxiras, R. C. Ashoori, and P. Jarillo-Herrero, Nature 556, 43 (2018); Y. Cao, V. Fatemi, S. Fang, S. L. Tomarken, J. Y. Luo, J. D. Sanchez-Yamagishi, K. Watanabe, T. Taniguchi, E. Kaxiras, R. C. Ashoori, and P. Jarillo-Herrero, Nature 556, 80 (2018). 


\title{
Weyl Semimetal Path to Valley Filtering in Graphene: Supplemental Material
}

\author{
Ahmed M. Khalifa, ${ }^{1}$ Ribhu K. Kaul, ${ }^{1}$ Efrat Shimshoni, ${ }^{2}$ H.A. Fertig, ${ }^{3}$ and Ganpathy Murthy ${ }^{1}$ \\ ${ }^{1}$ Department of Physics and Astronomy, University of Kentucky, Lexington, KY 40506-0055 \\ ${ }^{2}$ Department of Physics, Bar-Ilan University, Ramat-Gan 52900, Israel \\ ${ }^{3}$ Department of Physics, Indiana University, Bloomington, IN 47405
}

In this set of supplemental materials we present calculations substantiating statements made in the main text, and also present evidence of the robustness of valley-filtering to various perturbations to the highly symmetric model considered in the main text. That model retained the three-fold symmetry of the Hamiltonian in the hopping between the monolayer graphene (MLG) and the top layer of the Weyl semimetal (WSM). Further, that hopping was assumed to be spin-independent. In Section I we consider the most general hopping, which breaks the three-fold rotation symmetry and allows for spin flips during hopping. We present its effects on the spectrum in the $\mathbf{K}^{\prime}$ valley. In Section II we present numerical evidence for our main claim, that MLG states in the K valley "dissolve" into the bulk states of the WSM [S1]. In Section III we show the details of the calculation of the Landauer conductance of the $\mathbf{K}^{\prime}$ valley. Finally, in Section IV we treat a highly fine-tuned case, which is nevertheless quite interesting. This is the case when the energies of the MLG Dirac point and the WSM Weyl points coincide, and also lie at the chemical potential. A nontrivial reconstruction of the Fermi arcs takes place.

\section{GENERIC HOPPING BETWEEN GRAPHENE AND THE WSM SURFACE}

Having treated a highly symmetric model of hopping between the MLG and the top layer of the WSM in the main text (see Fig. 3 in the main text), we now treat the case where the $C_{3}$ symmetry is broken by the coupling. We also allow the spin to not be conserved in the tunneling process. We displace the two lattices with respect to each other to get a configuration where there is no center of rotation present (See Fig. S1(a)). There are 6 atoms per unit cell (at the interface) in our model; two of them being the A and the B sublattices in graphene and the remaining four belong to the WSM which we denote by numbers from 0 to 3 . The coupling will take the general form

$$
\left(\begin{array}{ll}
\kappa_{A, 0}(\vec{p}) & \kappa_{B, 0}(\vec{p}) \\
\kappa_{A, 1}(\vec{p}) & \kappa_{B, 1}(\vec{p}) \\
\kappa_{A, 2}(\vec{p}) & \kappa_{B, 2}(\vec{p}) \\
\kappa_{A, 3}(\vec{p}) & \kappa_{B, 3}(\vec{p})
\end{array}\right) \otimes\left(\begin{array}{cc}
\nu_{\uparrow \uparrow} & \nu_{\uparrow \downarrow} \\
\nu_{\downarrow \uparrow} & \nu_{\downarrow \downarrow}
\end{array}\right),
$$

where $\kappa_{A / B, i}(\vec{p})$ denotes the coupling between the A/B sublattice and the $i$ site of the WSM which is generally a function of momentum $\vec{p} . \nu_{\alpha \beta}$ denotes the coupling between spins $\alpha$ and $\beta$ in graphene and the WSM, respectively. Taking the configuration shown in Fig. S1(a) and keeping nearest neighbor terms only, (1.1) will take the form,

$$
\left(\begin{array}{cc}
\kappa_{A, 0} & \kappa_{B, 0} \\
0 & 0 \\
\kappa_{A, 2} e^{i \vec{p} \cdot \overrightarrow{b_{2}}} & \kappa_{B, 2} \\
\kappa_{A, 3} e^{i \vec{p} \cdot \overrightarrow{b_{1}}+i \vec{p} \cdot \overrightarrow{b_{2}}} & \kappa_{B, 3}
\end{array}\right) \otimes\left(\begin{array}{cc}
\nu_{\uparrow \uparrow} & \nu_{\uparrow \downarrow} \\
\nu_{\downarrow \uparrow} & \nu_{\downarrow \downarrow}
\end{array}\right)
$$

where $\overrightarrow{b_{1,2}}$ are the lattice bases vectors. The behavior of states in the $\mathbf{K}^{\prime}$ valley depends on the details of the coupling, as shown in Fig. S1(b),(c). No qualitative difference from the highly symmetric model of the main text is found in the spectrum near the $\mathbf{K}^{\prime}$ valley. We conclude that the spectrum presented in Fig. 4(c) of the main text is robust to making the tunneling matrix elements between the MLG and the WSM generic.

\section{DISSOLUTION OF GRAPHENE STATES INTO WEYL SEMIMETAL}

It is well-known that unless there are symmetry restrictions, or couplings are fine-tuned [S2], a localized state which is tunnel-coupled to a continuum of delocalized states will generically hyrbridize with them and dissolve into them [S1]. Here we present numerical evidence that there is no hidden symmetry/fine-tuning in our model that prevents this dissolution. 


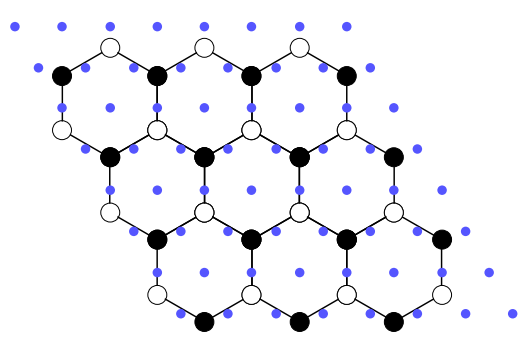

(a)

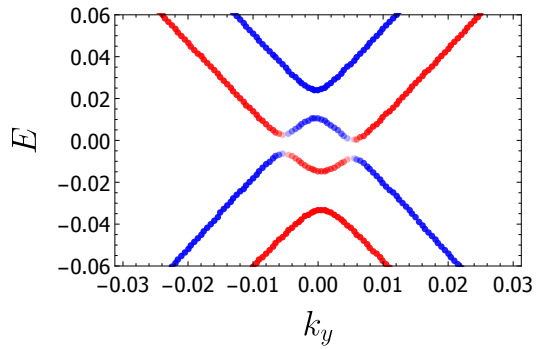

(b)

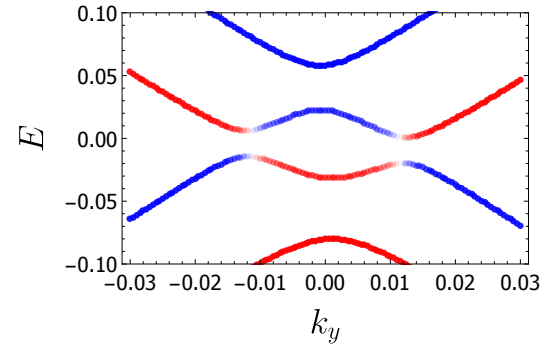

(c)

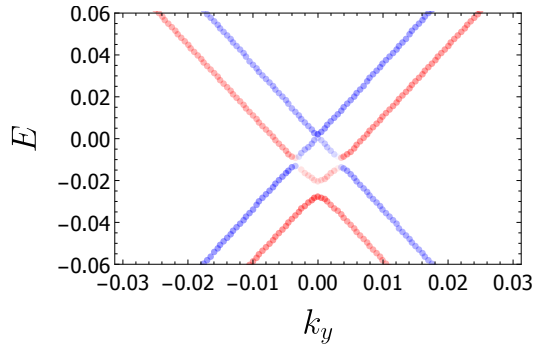

(d)

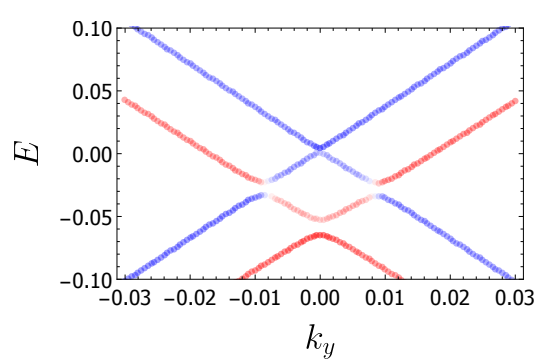

(e)

FIG. S1: General interface between graphene and the WSM. (a) Displacement of graphene and the WSM lattices lead to a structure with no $C_{3}$ symmetry present. (b),(c) A zoom-in on the $\mathbf{K}^{\prime}$ valley band structure with the tunneling conserving spin. (d),(e) A zoom-in on the $\mathbf{K}^{\prime}$ valley band structure with spin non-conserving processes present. The colors code the average of the spin operator's $z$ component with red being $\uparrow$ and blue being $\downarrow$. The dilution of the colors in (d),(e) implies less spin polarization. Values of the coupling in (b), (d) are $\kappa_{A, 0}=\kappa_{B, 2}=\kappa_{B, 3}=0.2, \kappa_{A, 2}=\kappa_{A, 3}=0.15$ and $\kappa_{B, 0}=0.1$. Values of the coupling in (c),(e) are $\kappa_{A, 0}=\kappa_{B, 2}=\kappa_{B, 3}=0.3, \kappa_{A, 2}=\kappa_{A, 3}=0.25$ and $\kappa_{B, 0}=0.2$.

To find the effect on a graphene state after the coupling is turned on, for a given value of $\vec{k}$ near the $\mathbf{K}$ valley, we look for states localized near the top surface, in a range of energies near that of the graphene unperturbed states. We use the inverse participation ratio $(I P R)$ as a measure for localization which is defined for a wave function $\psi$ as,

$$
I P R=\frac{1}{\sum_{i}\left|\psi_{i}\right|^{4}} .
$$

where $i$ is a joint index representing the layer, spin, and for graphene sites, sublattice as well. A completely localized state will give an IPR of order 1 whereas a completely delocalized state will give an IPR of order $N$, with $N$ being the dimension of the Hilbert space. Using this definition, we conduct a search for localized states at a momentum near the $\mathbf{K}$ valley and in the vicinity of the unperturbed energy. We then observe how the probability of the electron to be on the MLG behaves as the thickness of the WSM slab is increased and the bulk states become denser (See Fig. $\mathrm{S} 2 \mathrm{a})$. For different momenta near the $\mathbf{K}$ valley, one sees clearly that the states become delocalized as the the system size increases. One clearly sees the trend towards complete delocalization in the thermodynamic limit.

\section{CONDUCTANCE}

We next discuss how one calculates the conductance in a graphene-WSM device [S3], for which the result is given in Fig. 4(d) in the main text. We work within the effective model for the $\mathbf{K}^{\prime}$ valley introduced in equation (6) in the main text. In this simple model, there are four spin polarized bands and the spin carried by the bands alternates between up and down so it emulates the band inversion seen in the bands obtained from the tight binding calculation. In this model, however, the two middle bands touch at zero energy where there is a tiny gap in the bands obtained numerically. So as long as the chemical potential is away from this point (which is the regime we are interested in), the model should give qualitatively the same result as the numerical bands. 


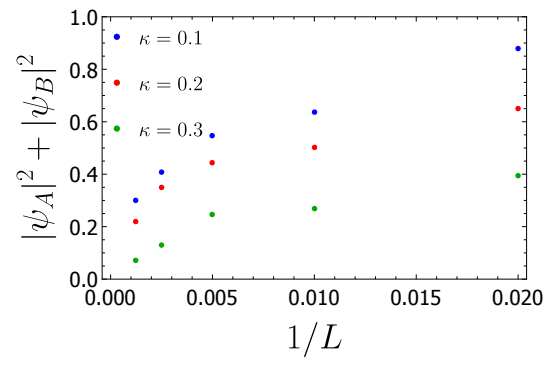

(a)

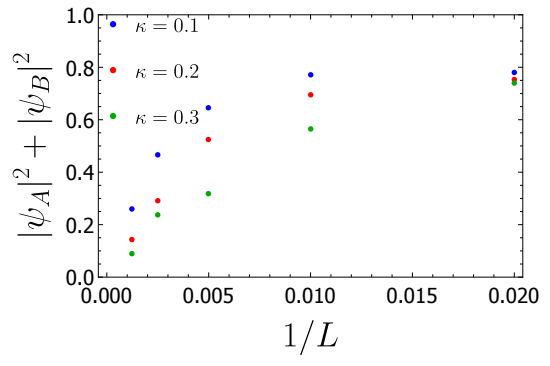

(b)

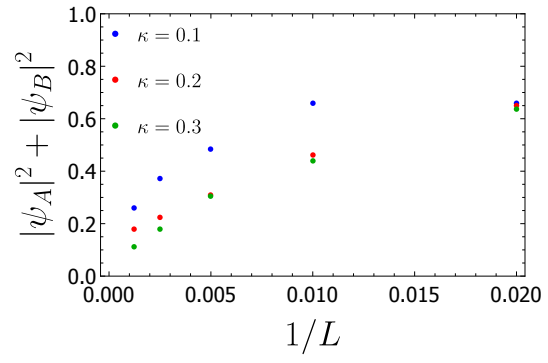

(c)

FIG. S2: Delocalization of the $\mathbf{K}$ valley. (a)-(c) The graphene wave function amplitudes as a function of the system size for momenta $\vec{p}=(2 \pi / 3,0), \vec{p}=(2 \pi / 3+0.025,0.014)$ and $\vec{p}=(2 \pi / 3-0.02,0.012)$ respectively. Calculations were done in the general configuration with the coupling in equation (1.2). $\kappa=0.1$ represents the set of parameters $\kappa_{A, 0}=\kappa_{B, 3}=0.1$ and $\kappa_{A, 2}=\kappa_{A, 3}=\kappa_{B, 0}=\kappa_{B, 2}=0.08 . \kappa=0.2$ represents the set of parameters $\kappa_{A, 0}=\kappa_{B, 3}=0.2$ and $\kappa_{A, 2}=\kappa_{A, 3}=\kappa_{B, 0}=\kappa_{B, 2}=0.18 . \kappa=0.3$ represents the set of parameters $\kappa_{A, 0}=\kappa_{B, 3}=0.3$ and

$$
\kappa_{A, 2}=\kappa_{A, 3}=\kappa_{B, 0}=\kappa_{B, 2}=0.28
$$

Looking at the schematic picture in Fig. 1 in the main text, we see that the strip is divided into three regions with graphene leads on the left $(x<0)$ and right $(x>L)$ at potential 0 while the middle region $(0<x<L)$ at potential $V_{g}$. We consider a scattering problem where we assume the potential to take the form

$$
V(x)= \begin{cases}0 & x \leq 0 \\ e V_{g} & 0 \leq x \leq L \\ 0 & L \leq x\end{cases}
$$

For the ease of notation we denote the x-momentum $k$ and the transverse momentum $q$. We also set $\hbar$ and graphene Fermi velocity $v$ to 1 . We show the conductance resulting from three different values for the chemical potential in Fig. S3 to show that the result obtained is not too sensitive to the chemical potential. We present the detailed calculation for the chemical potential $\mu_{1}$ in Fig. S3; the others can be obtained in a similar fashion.

To start, we need to find the dispersion of electrons in the leads and in the middle region. We have in the leads

$$
\varepsilon=\sqrt{k^{2}+q^{2}}
$$

For $0<x<L$, we have (using the continuum model of Eq. (6) in the main text)

$$
\varepsilon=e V_{g}-\lambda_{2}+\sqrt{\lambda_{1}^{2}+\tilde{k}^{2}+q^{2}},
$$

for the spin up band. For the spin down band, we have

$$
\varepsilon=e V_{g}+\lambda_{2}-\sqrt{\lambda_{1}^{2}+\tilde{k}^{2}+q^{2}}
$$

Note that $k$ in the leads is always real while $\tilde{k}$ can be imaginary.

Consider a scattering state coming from the left. We have for $x<0$,

$$
\Phi_{L}=\left(\begin{array}{c}
-1 \\
z_{k}
\end{array}\right) e^{i k x+i q y}+r\left(\begin{array}{c}
-1 \\
z_{-k}
\end{array}\right) e^{-i k x+i q y}
$$

For $0<x<L$, we have

$$
\tilde{\Phi}=\alpha\left(\begin{array}{c}
-1 \\
w_{\tilde{k}}
\end{array}\right) e^{i \tilde{k} x+i q y}+\beta\left(\begin{array}{c}
-1 \\
w_{-\tilde{k}}
\end{array}\right) e^{-i \tilde{k} x+i q y}
$$

For $x>L$, we have

$$
\Phi_{R}=t\left(\begin{array}{c}
-1 \\
z_{k}
\end{array}\right) e^{i k(x-L)+i q y}
$$


$z_{k}=\frac{k-i q}{\sqrt{k^{2}+q^{2}}} \cdot w_{\tilde{k}}=\frac{\tilde{k}-i q}{\lambda_{1}+\sqrt{\lambda_{1}^{2}+\tilde{k}^{2}+q^{2}}}$ for the spin up states, and $w_{\tilde{k}}=\frac{\tilde{k}-i q}{\lambda_{1}-\sqrt{\lambda_{1}^{2}+\tilde{k}^{2}+q^{2}}}$ for the spin down states. $r$ and $t$ define the reflection and the transmission probabilities, respectively.

Using the continuity of the wave functions at $x=0$ and $x=L$, we can solve for the transmission amplitude $t$, resulting in

$$
t=\frac{\left(1+z_{k}^{2}\right)\left(w_{\tilde{k}}-w_{-\tilde{k}}\right)}{e^{i \tilde{k} L}\left[\left(1+z_{k} w_{-\tilde{k}}\right)\left(w_{\tilde{k}}-z_{k}\right)\right]+e^{-i \tilde{k} L}\left[\left(1+z_{k} w_{\tilde{k}}\right)\left(z_{k}-w_{-\tilde{k}}\right)\right]}
$$

The transmission probability is given by $T=|t|^{2}$. Plotting this equation as a function of the conserved transverse momentum $\left(k_{y}\right)$ gives Fig. $(4)(\mathrm{d})$ in the main text.

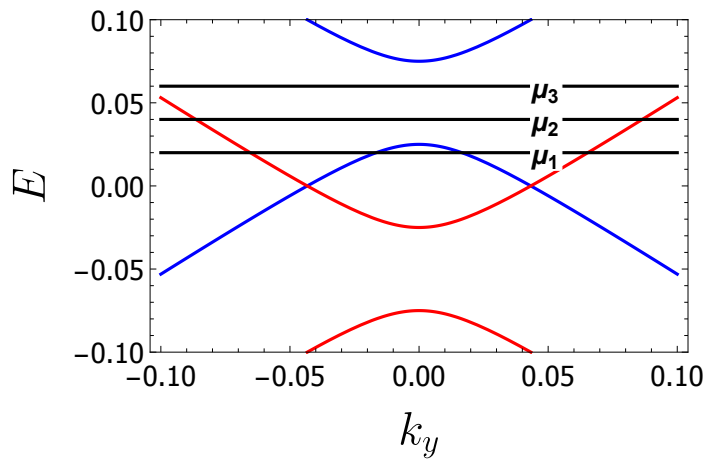

(a)

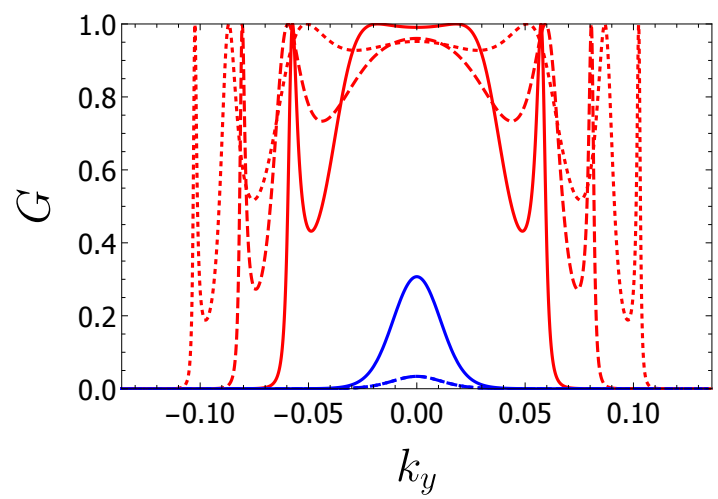

(b)

FIG. S3: Conductance at different chemical potentials. (a) The bands obtained from the continuum model of equation (6) in the main text. The three horizontal black lines represent three values for the chemical potential. (b) The conductance measured in units of $\left(e^{2} / h\right)$ for the three respective chemical potential. Solid, $\mu_{1}=0.02$, Dashed, $\mu_{2}=0.04$ and Dotted, $\mu_{3}=0.06$. In (a),(b) red denotes spin $\uparrow$ and blue denotes spin $\downarrow$.

\section{GRAPHENE-FERMI ARC HYBRIDIZATION}

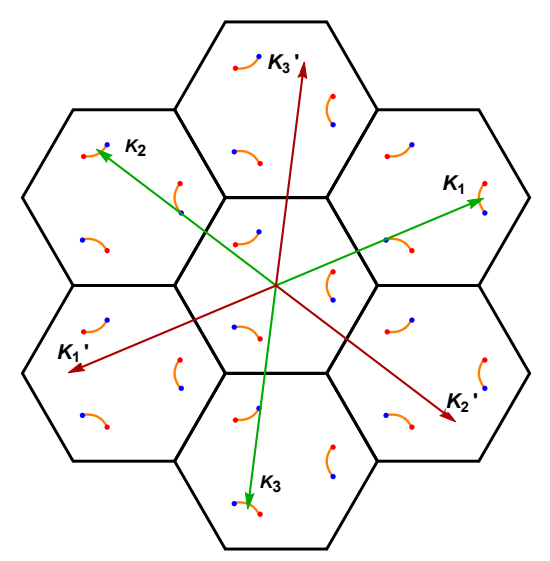

FIG. S4: an incommensurate structure of graphene and the WSM shown in momentum space. The 3 equivalent graphene $\mathbf{K}$ vectors coincide with the Fermi arcs.

Another possibility that this system offers is the hybridization between graphene's Dirac cones and the Fermi arc states localized on the surface of the WSM. This again will occur in only one of graphene's valleys due to the breaking of time reversal symmetry. This construction, however, requires some fine-tuning as we need the chemical 
potential to be at the Dirac points in graphene and at the Weyl nodes in the WSM. Furthermore, we need the $K$ point in graphene to coincide with the Fermi arcs as shown in Fig. S4 which happens at certain orientations of the graphene-WSM system.

In a fashion similar to our treatment in the main text, we consider a commensurate structure of graphene and the WSM with the Dirac $\mathbf{K}$ point overlapping a Fermi arc in momentum space. We then perform our tight binding calculations to study the electronic properties of this system. A plot for the band structure near the $\mathbf{K}$ valley shows the effect of the WSM Fermi arc on the Dirac cone is shown in Fig. S5(a).

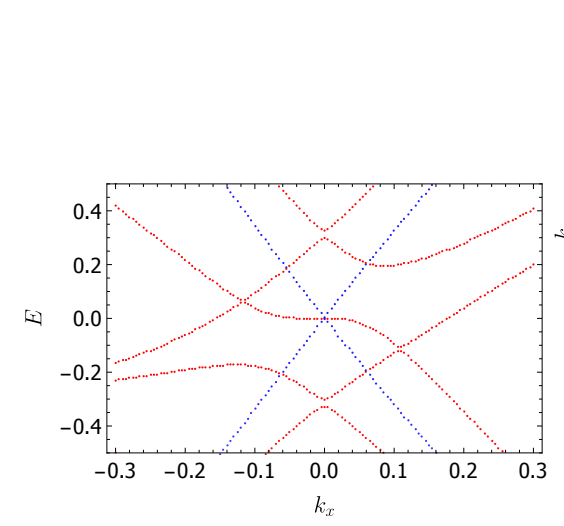

(a)

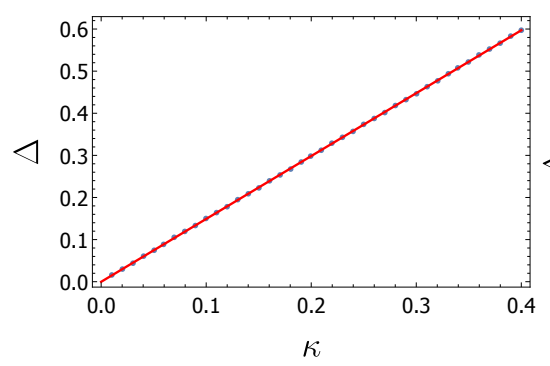

(c)

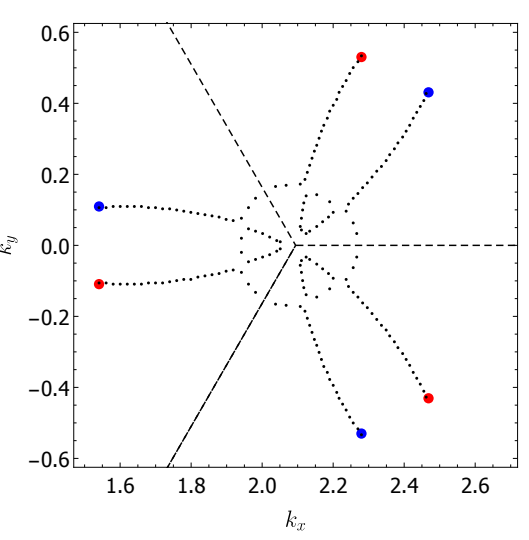

(b)

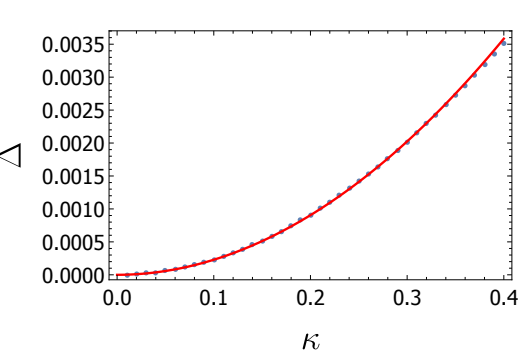

(d)

FIG. S5: Fermi Arcs hybridization with the $\mathbf{K}$ valley. (a) The Dirac cone near the $\mathbf{K}$ valley along the $k_{x}$ axis in momentum hybridizing with Fermi arc states present on the top surface of the WSM. (b) A plot of the reconstructed Fermi arcs near the $\mathbf{K}$ point which shows the detachement of the arcs into three closed loops. (c) The gap in the spin $\uparrow$ Dirac cone scales linearly with the coupling as the data points fits nicely to a straight line (in red). (d) The gap in the spin $\downarrow$ Dirac cone scales quadratically with the coupling as the data is fitted nicely to a parabola (in red).

We can understand the resulting band structure based on the fact that the Fermi arc states are spin polarized in our simple model with the spins being up for the top WSM surface. Consequently, the spin up Dirac cone acquires a gap which is shown to scale linearly with the coupling as shown in Fig. S5(c). The spin down cone, however, remains relatively intact (See Fig. S5(a)) but upon careful examination, we can see it acquires a small gap which scales quadratically with the coupling as shown in Fig. S5(d).

Finally, looking at the zero energy contour, we can see a reconstruction of the Fermi arcs as shown in Fig. S5(b). The existence of a Fermi loop at the surface can lead to interesting resonance signatures in the optical conductivity of the system in a magnetic field perpendicular to the surface.

[S1] G. D. Mahan, Many Particle Physics, Third Edition (Plenum, New York, 2000).

[S2] Hsu, C., Zhen, B., Stone, A. et al. Bound states in the continuum. Nat Rev Mater 1, 16048 (2016).

[S3] Tworzydło, J., Trauzettel, B., Titov, M., Rycerz, A. and Beenakker, C., Phys. Rev. Lett. 96 (2006). 